\title{
O que as pesquisas em educação matemática apontam sobre estimativas numéricas de crianças?
}

\author{
What does mathematical education research points about number estimation of \\ children?
}

\author{
Mariana Lima Duro ${ }^{1}$ \\ Beatriz Vargas Dorneles ${ }^{2}$
}

\begin{abstract}
Resumo
Trata-se de uma revisão bibliográfica acerca do conhecimento produzido sobre estimativa numérica nas últimas três décadas, com objetivo de destacar a sua importância enquanto habilidade matemática. Observou-se que estão sendo discutidos cinco aspectos principais: as suas possíveis bases mentais, a sua relação com a representação mental dos números, o uso de estratégias e as maneiras de avaliar o seu desempenho (julgamento de quantidades e estimativa na reta numérica). Os resultados indicam que os sujeitos possuem diferentes formas de representação numérica mental e de estratégias de resolução de tarefas envolvendo estimativa numérica, demonstrando que o processo estratégico é dinâmico e construído pelo sujeito de acordo com os fatores envolvidos na situação apresentada. Conclui-se que a estimativa numérica é considerada, pelos estudos revisados, uma habilidade matemática necessária para o sucesso na vida adulta, devendo, portanto, ter espaço de discussão na escola.
\end{abstract}

Palavras-chave: Estimativa numérica; Estimativa na reta numérica; Estratégias de estimativa.

\begin{abstract}
It is a bibliographical review about the knowledge produced on number estimation in the last three decades, in order to highlight its importance as a mathematical ability. It was observed that five main aspects are being discussed: their possible mental bases, their relation with the mental representation of numbers, the use of strategies and the ways of evaluating their performance (numerosity and number line estimation). The results indicate that the subjects have different forms of mental numerical representation and task resolution strategies involving number estimation, demonstrating that the strategic process is dynamic and that it is constructed by the subject according to the factors involved in the presented situation. It is concluded that the number estimation should be considered a mathematical skill necessary for success in adult life, having space for discussion in the school.
\end{abstract}

Keywords: Number estimation; Number Line estimation; Estimative strategies.

Submetido em: 04/07/2018 - Aceito em: 20/12/2019 - Publicado em: 25/12/2019

${ }^{1}$ Doutora em Educação pela Universidade Federal do Rio Grande do Sul. Professora e coordenadora do Curso Superior de Licenciatura em Matemática do Campus Canoas do Instituto Federal de Educação, Ciência e Tecnologia do Rio Grande do Sul, Brasil. mariana.duro@ canoas.ifrs.edu.br. OCID ID: https://orcid.org/00000001-5492-4098.

${ }^{2}$ Doutora em Psicologia Escolar e do Desenvolvimento Humano na Universidade de São Paulo. Professora titular do Departamento de Estudos Especializados da Faculdade de Educação da Universidade Federal do Rio Grande do Sul e do Programa de Pós-graduação em Educação da mesma universidade. ORCID ID: https://orcid.org/0000-0002-0141-9140. 


\section{Introdução}

Na década de 80 já eram ressaltadas as tentativas de se esclarecer quais as habilidades matemáticas seriam necessárias para o sucesso na vida adulta (Levine, 1982). Ainda nesta época, a capacidade de realizar estimativas numéricas mentais aparecia em muitas dessas listas, destacando-se sua utilidade em situações cotidianas que envolvem números, inclusive para julgar se o resultado exato obtido é razoável para o seu propósito. Antes disso (Levine, 1982; Siegel, Goldsmith \& Madson, 1982), não há indícios de pesquisas que tenham sido dedicadas a compreender aspectos cognitivos referentes à capacidade de realizar estimativas numéricas, mesmo que, de fato, essa capacidade já venha se desenvolvendo e tendo um grande papel na construção do conhecimento matemático desde a antiguidade.

Sabe-se que o sistema de contagem decimal, utilizado em diversos países, incluindo o Brasil, estabelece uma quantificação exata das quantidades, permitindo reconhecer diferenças precisas, diferentemente do que acontece quando se realizam estimativas. No entanto, crianças e adultos, mesmo possuindo estas representações exatas, também utilizam estimativas durante toda sua vida (Feigenson, Libertus \& Halberda, 2013), tendo em vista que a capacidade de realizar estimativas com razoável precisão torna a vida mais fácil (Siegler \& Booth, 2004) e, em determinados contextos, é mais conveniente que a quantificação exata (Azevedo, 1996).

Estimativa numérica é um processo de tradução entre representações quantitativas alternativas, nas quais pelo menos uma não é exata e, pelo menos, uma é numérica (Siegler \& Booth, 2005). Por exemplo, a estimativa na reta numérica requer traduzir um número em uma posição espacial ou traduzir uma posição espacial em um número. Da mesma forma, a estimativa de quantidades requer traduzir uma representação quantitativa de objetos nãonumérica em um número e a estimativa computacional envolve a tradução de uma representação numérica exata (por exemplo, $75 \times 29$ ) para uma inexata (cerca de 2200).

Assim, a habilidade de realizar estimativas pode ser extremamente relevante na resolução de tarefas matemáticas, tendo em vista que, para sua compreensão, é preciso entender tanto as magnitudes reais quanto as relativas dos números e, saber realizar estimativas plausíveis, pode contribuir para que não ocorra uma compreensão limitada ou insuficiente dos números. Entretanto, apesar da reconhecida importância do tema, o que se observa é que a estimativa numérica foi pouco investigada em comparação à contagem exata (Rousselle \& Noel, 2008). Sendo assim, ainda é pouco claro se a proficiência em estimativa poderia ser uma das causas de maior habilidade matemática ou o contrário.

Para compreender melhor essas questões, é necessário discutir as teorias que discorrem sobre o tema e de que forma a maneira como entendem o aprendizado encaminham o método e influenciam os resultados das pesquisas em educação. Nesse sentido, este estudo visa realizar uma revisão bibliográfica, a partir da base de dados disponibilizada pela Coordenação de Aperfeiçoamento de Pessoal de Nível Superior (CAPES), acerca do tema da estimativa numérica, considerando os estudos publicados na área de educação matemática e destacando estudos sobre as habilidades cognitivas consideradas bases mentais que originam 
esta habilidade, estudos sobre a relação entre estimativa numérica e a representação mental das magnitudes numéricas, estudos sobre as diferentes maneiras de avaliar o desempenho em estimativa, estudos que comparam o desempenho em tarefas de estimativa numérica ao desempenho em matemática, bem como, estudos que evidenciam o uso de diferentes estratégias para realizar estimativas.

Para encontrar textos que discorriam sobre os assuntos citados anteriormente, buscouse artigos publicados em periódicos indexados, em língua portuguesa e inglesa. Observa-se que, até a presente data, somente um texto em língua portuguesa foi encontrado, mas este não se trata de pesquisa brasileira, mas portuguesa. Foi realizada uma busca por assunto, cujas palavras-chave utilizadas foram "estimativa numérica", "estimativa na reta numérica", "estimativa de quantidades" e "estratégia de estimativa", bem como as keywords correspondentes na língua inglesa: "numerical/number estimation", "number-line estimation, "numerosity" e "estimation estrategy". Não houve limitação temporal na pesquisa, sendo as primeiras evidências encontradas a partir da década de 1980.

Esta pesquisa integra um projeto mais abrangente, intitulado "Diversidade na aprendizagem da matemática inicial: a compreensão da estimativa numérica" (Plataforma Brasil e Comitê de Ética em Pesquisa da Universidade Federal do Rio Grande do Sul, sob o número 31575913.6.0000.5347). O projeto tem como proposta o estudo da diversidade na aprendizagem da matemática, no que se refere à compreensão da estimativa numérica em diferentes grupos de alunos. Assim, para compreender o processo de construção da estimativa numérica nos sujeitos, organizou-se a presente pesquisa em cinco capítulos de discussão teórica, que serão descritos na sequência.

O primeiro capítulo discute as possíveis bases cognitivas da estimativa numérica, identificando os indícios de que essa capacidade é construída pelo sujeito ao longo da vida. Em seguida, o segundo capítulo discorre sobre os modelos de representação numérica mental identificando diferentes processos que explicam a construção da estimativa numérica, destacando processos que indicam que esses modelos podem não ser únicos e que podem estar presentes em diferentes etapas do desenvolvimento do sujeito. No terceiro capítulo, trazse estudos sobre estratégias de estima numérica, demonstrando que o processo estratégico é dinâmico e que é construído pelo sujeito de acordo com os fatores envolvidos na situação apresentada. Assim, no quarto e no quinto capítulo do referencial teóricos, discorre-se sobre os estudos que enfatizam os principais testes utilizados na avaliação de desempenho em estimativa numérica: estimativa numérica de quantidades discretas e estimativa na reta numérica, respectivamente. Discutir sobre esses testes permite-nos identificar de que forma o processo de construção da estimativa numérica ocorre no sujeito e se é semelhante para os diferentes tipos de testes adotados pelos pesquisadores. Dessa forma, pretende-se abranger os diferentes aspectos abordados pela literatura atual, considerando o tema da estimativa numérica. 


\section{Possíveis bases cognitivas da estimativa numérica}

A competência matemática compreende uma grande variedade de diferentes habilidades e processos cognitivos e algumas habilidades matemáticas elementares, como a discriminação de pequenas quantidades ou cálculos aritméticos com pequenos conjuntos de objetos são observados em crianças (Starkey \& Cooper, 1980). Esses achados foram interpretados por alguns pesquisadores como evidências de que o cérebro humano seria dotado de um senso numérico inato (Dehaene, 1997).

Ainda anteriormente à aquisição da quantificação exata, alguns pesquisadores consideram a existência de uma capacidade de enumerar pequenas quantidades de 3 ou 4 itens apresentadas por curtos períodos de tempo (Dehaene, 1997), chamada de "subitizing". Apesar da concordância geral entre os pesquisadores de que a enumeração é tratada de forma diferente dentro do intervalo de subitizing, tem havido algum debate sobre se o subitizing utiliza mecanismos iguais ou diferentes para a estimativa de intervalos numéricos maiores.

Os resultados de Libertus, Feigenson e Halberda (2011), que mediram a acurácia do Sistema de Aproximação Numérica (ANS) de crianças, forneceram evidências de que uma relação entre o senso numérico primitivo e a capacidade matemática começa desde o nascimento, apoiando a noção de uma ligação estreita entre um senso numérico primitivo e habilidades matemáticas formais. Park e Brannon (2013) também encontram correlação entre a acuidade da ANS e desempenho em matemática simbólica, mostrando que a adição e subtração aproximadas de matrizes de pontos melhoram a adição e subtração simbólicas. Tal pressuposto sustenta a hipótese de que as habilidades matemáticas complexas são fundamentalmente ligadas às habilidades quantitativas pré-verbais.

Ao contrário, Gebuis e Reynvoet (2012) propõem que os seres humanos estimam quantidades através da ponderação dos diferentes elementos visuais presentes nos estímulos. Assim, esta não seria uma capacidade quantitativa propriamente dita e estaria relacionada com fatores viso-espaciais. Halberda e Feigenson (2008) investigaram o ANS em crianças e adultos com duas matrizes contendo entre 1 e 14 itens para ser escolhida a matriz que apresentava a maior quantidade de itens. Eles mostraram que essa habilidade continua a crescer durante toda a infância, com níveis de acurácia atingidos surpreendentemente tarde no desenvolvimento, não antes do início da adolescência. Os autores destacam que o ANS é observado em crianças desde muito antes do ensino de matemática simbólica, por isso, pode ter um papel causal no desempenho matemático individual e que a capacidade de raciocinar sobre números simbólicos está profundamente entrelaçada com ANS. Ou seja, para estes pesquisadores, a capacidade de realizar estimativas de quantidades vai além do senso numérico (no sentido inato) e se desenvolve com a idade e com a experiência.

Pesquisadores como Verschaffel e colaboradores (1998), embora afirmem que a estimativa e a quantificação exata não sejam caminhos opostos e que podem estar estreitamente relacionadas, tal como pensam os construtivistas, consideram diversos fatores da Psicologia Cognitiva em suas constatações sobre o desenvolvimento da estimativa 
DOI: $10.20396 /$ zet.v27i0.8652389

numérica. Essa perspectiva dialógica parece ser interessante, tendo em vista que a capacidade matemática pode ter uma origem biológica, mas seu desenvolvimento depende de fatores externos e das interações que possam ocorrer na vida do sujeito para a aquisição de conhecimentos mais complexos.

A origem do SN ainda é um ponto divergente entre os pesquisadores. Corso e Dorneles (2010) consideram que o SN é construído e que está relacionado à interação com números e ao desenvolvimento de estratégias. Em contrapartida, a ciência cognitiva trata o SN como uma capacidade inata localizada no sistema neuronal juntamente com a quantificação exata (Dehaene, 1997). Para integrar essas duas concepções, Berch (2005) propõe uma perspectiva que aceita uma possível base orgânica para capacidade numérica das pessoas, mas que esta base não impossibilitaria uma construção deste sistema posteriormente, através das experiências. É nesta perspectiva de diálogo que foram discutidos os achados desta pesquisa.

\section{Modelos de representação numérica mental}

Numa série de estudos, Siegler e colaboradores (Booth \& Siegler, 2006; Laski \& Siegler, 2007; Siegler \& Booth, 2004; Siegler \& Opfer, 2003) indicaram que, durante os primeiros anos de escolaridade, o conhecimento das crianças sobre magnitudes numéricas sofre alterações fundamentais. O pressuposto de que o conhecimento numérico das crianças durante esses anos não apenas cresce quantitativamente, mas, acima de tudo, que os padrões subjacentes de representações numéricas sofrem uma mudança qualitativa, foi investigado em diferentes tarefas de estimativa. Alguns autores sugerem que as representações mentais de número são realizadas por meio de uma reta numérica mental (Laski \& Siegler, 2007; Link, Nuerk \& Moeller, 2014) e que a tradução de números em posições na reta numérica está relacionada a representações mentais de magnitudes numéricas.

Para isso, uma das tarefas amplamente utilizadas para compreender a representação mental dos números é a tarefa de estimativa na reta numérica (Barth \& Paladino, 2011; Dehaene et al., 2008; Ebersbach et al., 2008; Moeller et al., 2009; Muldoon et al., 2011; Siegler \& Booth, 2004; Siegler \& Opfer, 2003). Os dados empíricos coletados a partir da tarefa de estimativa na reta numérica levaram pesquisadores a proporem vários modelos de representação numérica mental. Esses modelos são descritos, a seguir, com o intuito de compreender de que forma essa representação vem sendo discutida.

\section{Modelo Acumulador ou Linear}

Em um primeiro momento, Gibbon e Church (1981) propõem um modelo em que a distância entre os números posicionados na reta numérica mental seria constante e linear. Ou seja, que a razão entre a variabilidade da estimativa e a magnitude a ser estimada mantém-se constante (variabilidade escalar), aumentando a imprecisão conforme aumentam as magnitudes a serem representadas. Do mesmo modo, a representação de grandezas de magnitudes próximas seria mais difícil de ser discriminada do que a representação de 
grandezas mais afastadas.

\section{Modelo Logarítmico}

Ao contrário do modelo anterior, esse modelo sugere que a percepção de quantidade aumenta logaritmicamente, preservando as relações de razão, em vez de diferenças absolutas (Dehaene, 1997). Ou seja, exagera-se a distância entre os números pequenos e minimizam-se a distância entre médios e grandes números. Como um caso extremo, Dehaene e colaboradores (2008) ainda encontraram evidências, em um estudo realizado na Amazônia, da presença de tal modelo em adultos, sem linguagem matemática estruturada, apoiando a ideia de que a representação logarítmica é o caminho inicial e intuitivo de mapeamento numérico.

Siegler e Opfer (2003) sugeriram um modelo que concilia os dois modelos descritos anteriormente, prevendo uma possível mudança logarítmico-linear, baseada nas habilidades quantitativas das crianças. Este modelo conciliador sugere que a representação logarítmica é dominante no início do desenvolvimento e, com a idade, a experiência e o conhecimento de um intervalo numérico cada vez maior, a representação tornar-se-ia linear. Concluíram também que, embora a representação das crianças possa ser melhor descrita por um modelo linear para intervalos numéricos menores, ela ainda pode ser logarítmica para intervalos maiores (Siegler \& Opfer, 2003; Siegler \& Booth, 2004).

$\mathrm{O}$ apoio mais forte para a hipótese de mudança logarítmica-linear veio de um grande número de estudos realizados por Siegler e seus colegas (Booth \& Siegler, 2006, 2008; Laski \& Siegler, 2007; Siegler \& Booth, 2004; Siegler \& Mu, 2008; Siegler \& Opfer, 2003). Eles identificaram diferentes idades em que as crianças americanas se afastam de representações logarítmicas e desenvolvem representações lineares para diferentes intervalos numéricos. Especificamente, eles descobriram que a linearidade no intervalo numérico de 0-10 foi atingida por pré-escolares, que a linearidade na faixa de 0-100 foi atingida por alguns alunos da $1^{\mathrm{a}}$ e $2^{\mathrm{a}}$ séries, e que a linearidade na faixa 0-1000 foi atingida por alunos da $4^{\mathrm{a}}, 5^{\mathrm{a}}$ e $6^{\mathrm{a}}$ séries.

Tanto o modelo logarítmico quanto o modelo acumulador (linear) fornecem dados de como as pessoas representam quantidades. No entanto, parece provável as crianças conhecerem e usarem várias representações de quantidades numéricas e que o contexto numérico influencie a escolha da representação utilizada. Possuir múltiplas representações é útil porque podem ser igualmente boas em situações diferentes, sendo a mudança de representação (de logarítmicas para linear) importante por razões teóricas e empíricas.

\section{Modelo Duplo-linear}

Com base nas representações numéricas de números de dois dígitos, Moeller e colaboradores (2009) propuseram um modelo representado por duas ou mais funções lineares. Nesse modelo, a representação mental de números poderia ser descrita, alternativamente, por uma combinação de dois padrões lineares com diferentes inclinações. Nuerk e colaboradores (2004) constaram que as dezenas e as unidades de números de dois 
dígitos são processadas separadamente (da esquerda para a direita) e não em sua totalidade. A explicação dos autores para este achado estaria no fato de que as crianças demorariam mais tempo a fazer comparações quando o dígito da unidade em um número é maior do que o dígito da dezena (por exemplo, 19 e 91). Os autores argumentam que os números são decompostos por seus dígitos separadamente.

A proposição de um modelo linear de duas fases lança uma ideia diferente sobre os resultados de Siegler e colaboradores (Booth \& Siegler, 2006; Siegler \& Booth, 2004; Siegler \& Opfer, 2003) em relação à sua reivindicação de múltiplas representações mentais de número, sugerindo que pode haver apenas um tipo de representação em cada indivíduo, que consiste em, pelo menos, dois segmentos lineares, descartando a hipótese de múltiplas representações.

\section{Modelo de Familiaridade com Números}

Considerando as mudanças de conhecimento da magnitude numérica das crianças, Lipton e Spelke (2005) descreveram um modelo que sugere que as estimativas dos números familiares são mais precisas do que as de números desconhecidos, fornecendo apoio empírico a favor da hipótese de que a familiaridade com números leva a estimativas lineares. Ebersbach e colaboradores (2008) constaram que as crianças que contavam melhor tinham suas estimativas mais precisas. Sendo assim, a familiaridade das crianças com os números refletiria diretamente na sua reta numérica mental, sendo esta linear, contanto que os números estejam dentro do intervalo de números familiares às crianças (Ebersbach et al., 2008).

\section{Modelo de Juízo Proporcional}

Mais recentemente, Barth e Paladino (2011) propuseram as versões do modelo de juízo proporcional de um e de dois ciclos para dar conta da mudança de representação logarítmica-linear. O modelo de um ciclo considera a estratégia que utiliza somente os pontos de origem e o ponto final da reta, enquanto o modelo de dois ciclos sugere que as crianças dependem de ambos os pontos, inicial e final, mais o ponto médio, o que leva a um modelo de dois ciclos. Estes modelos indicam que as estimativas são imprecisas longe dos pontos de início e fim e de outros pontos de referência conhecidos. Siegler e Booth (2004) já haviam discutido anteriormente a importância dessas marcações, mas Barth e Paladino (2011) trazem a ideia de que a reta não é uma representação pura, mas que pode estar influenciada pelo conhecimento numérico.

Considera-se bastante consistente a ideia de complementariedade entre os modelos (Dorneles et al., 2017). Recentemente, Dackermann e colaboradores (2015) propuseram essa integração, sugerindo que a cada modelo possa representar a compreensão das magnitudes numéricas, dependendo da idade do sujeito e da sua familiaridade com o intervalo numérico (Siegler \& Booth, 2004; Booth \& Siegler, 2006).

Embora grande parte das pesquisas sobre as representações numéricas mentais tenha se centrado na busca de padrões de desenvolvimento únicos, alguns estudos também 
examinaram se o desempenho variaria de acordo com as origens culturais. Siegler e $\mathrm{Mu}$ (2008) constataram que a estimativa na reta numérica 0-100 de pré-escolares americanos era melhor explicada por uma função logarítmica, diferentemente de seus pares chineses, cujas estimativas eram lineares. Curiosamente, quando crianças chinesas foram pareadas no desempenho aritmético com crianças escocesas, Muldoon e colaboradores (2011) constataram que suas estimativas não eram mais lineares do que as crianças escocesas mais velhas.

\section{Estudos de estratégia em estimativa numérica}

Ainda na década de 80, Siegel, Goldsmith e Madson (1982) investigaram a capacidade de estimativa de medição de crianças entre 7 e 8 anos e jovens adultos a partir da decomposição em pequenas amostras de medida conhecida e recomposição do todo. Encontraram fraca relação entre a precisão da estimativa e o uso de estratégias, mas uma forte relação entre estimativa e o conhecimento das crianças sobre sistemas de medida, números e cálculos. Os pesquisadores elencaram algumas estratégias de resolução de problemas de estimativa: a) estratégia baseada na capacidade perceptiva, b) estratégia baseada na referência de um padrão conhecido, c) o valor de referência fracionado e múltiplo, correspondente à utilização de um padrão e d) a estratégia de decomposição/recomposição. Na década seguinte, Crites (1992) ampliou os achados de Siegel, Goldsmith e Madson (1982) identificando que estimadores bem-sucedidos tenderam a usar múltiplas estratégias e estimadores não tão bem-sucedidos geralmente usavam estratégias perceptivas.

Com foco nas mudanças de estratégias, Lemaire e Siegler (1995) realizaram um estudo que constatou que crianças da $2^{\mathrm{a}}$ e da $6^{\mathrm{a}}$ série não diferem no tipo de estratégia de estimativa utilizada, mas diferem na eficiência com que as aplicavam. Os pesquisadores chamaram essa capacidade de julgar a melhor estratégia de acordo com a situação de "competência estratégica" e distinguiram quatro situações que podiam influenciar na velocidade e na precisão da resposta obtida: a) aquisição de novas estratégias e abandono das antigas, b) utilização com maior frequência de estratégias mais eficientes disponíveis, c) melhoria na fluência e eficiência com que as estratégias são executadas e d) melhoria na capacidade de adaptação no momento da escolha de estratégia.

Como um meio de obter características das estimativas, Siegler e Lemaire (1997) introduziram o método de escolha/não-escolha. Esse método é baseado na possibilidade de a criança escolher uma estratégia para solucionar um conjunto de problemas, sendo que os pesquisadores avaliam a estratégia usada em cada problema, calculam a velocidade média e a precisão para cada estratégia ou sugerem uma estratégia específica que deve ser utilizada na solução dos problemas. Em 2009, Luwel e colaboradores (2009) apresentaram uma discussão sobre o método de escolha/não escolha, afirmando que este sofre de dois problemas graves: 1) os resultados e conclusões sobre a eficiência da estratégia são influenciados por efeitos de seleção, que podem envolver tanto o tipo de problema em que as estratégias são utilizadas, 
quanto as diferenças individuais de preferência de estratégia; 2) este método não permite estudar a adaptabilidade das escolhas estratégicas das pessoas.

No estudo de Verschaffel e colaboradores (1998), estudantes da $2^{\mathrm{a}}$ e $6^{\mathrm{a}}$ série e alunos universitários realizaram estimativas da quantidade. De acordo com os pesquisadores, pelo menos duas diferentes estratégias deveriam ser utilizadas: adição dos grupos e subtração da quantidade de quadrados vazios do total de quadrados da matriz. Seus resultados indicaram que a escolha entre a estratégia da adição ou da subtração é altamente determinada pela quantidade de blocos apresentados na matriz. Mais especificamente, os participantes geralmente escolhem a estratégia de adição nas tarefas em que a matriz contém poucos blocos preenchidos, enquanto adotam a estratégia de subtração nas tarefas que contém uma grande quantidade de blocos, com poucas células vazias. A partir da observação do tempo e da precisão das respostas de cada sujeito, os pesquisadores consideraram que a utilização mista e adaptativa das estratégias aumentaria com a idade, convergindo para evidências de uma tendência para a utilização da estratégia de subtração como a mais eficiente.

Não considerando suficientes as duas estratégias encontradas por Verschaffel e colaboradores (1998), Luwel e colaboradores (2000) investigaram o desenvolvimento das estratégias em estimativa de quantidades, encontrando mais um grupo de estratégias: a) adição dos grupos de blocos, b) subtração do número de quadrados vazios da quantidade total de blocos e c) uma estimativa grosseira determinada de modo rápido, mas impreciso. Ou seja, encontraram uma terceira estratégia, não antes relatada, utilizada em diferentes situações: tanto em matrizes com maior quantidade de estímulos a serem contados quanto aquelas com menor quantidade de estímulos.

Mesmo que três diferentes estratégias já tivessem sido anteriormente descritas em 2000, em 2001 Luwel e colaboradores (2001) propuseram-se a identificar estratégias e mudanças de estratégias em tarefas de julgamento de quantidades, já que o desempenho em uma tarefa não depende apenas da eficiência com que as estratégias disponíveis são executadas, mas também dos motivos que geram mudanças do uso de determinada estratégia e da frequência e da adaptabilidade com que as estratégias disponíveis são executadas. Os autores descreveram três modelos: a) o modelo de uma fase, que corresponde à estratégia de adição para todos os itens; b) o modelo de duas fases, que corresponde ao uso da estratégia da adição para pequenas quantidades de itens e da subtração para grandes quantidades e c) o modelo de três fases, que corresponde a uma estimativa rápida, influenciada pela informação sobre o tamanho da matriz. O modelo de duas fases aumentou com a idade, enquanto que o de uma fase diminuiu com a idade. Além disso, foi encontrada uma relação forte e positiva entre o uso da estratégia de subtração e precisão. Os alunos da $6^{\mathrm{a}}$ série foram mais precisos do que os alunos de $2^{\text {a }}$ série apenas quando eles não receberam informação sobre o tamanho da matriz.

Continuando suas pesquisas sobre estratégias de estimativa de quantidades, Luwel e Verschaffel (2003) investigaram o efeito da pressão do tempo sobre as estratégias de estimativa. $\mathrm{O}$ repertório de estratégias dos participantes e a frequência e eficiência de 
utilização das estratégias foram afetados pelo tempo, afetando a precisão geral dos estudantes. Os autores observaram uma diminuição não significativa na capacidade de adaptação com o aumento da pressão do tempo, embora tenha havido uma tendência nessa direção.

Com sua constante busca para compreender as estratégias de estimativa, Luwel e Verschaffel (2008) discutiram três estudos que consideravam o modelo de análise de Lemaire e Siegler (1995) para a utilização de estratégias no contexto de uma tarefa de julgamento de quantidades. Uma observação interessante foi que o fornecimento de feedback sobre as escolhas de estratégia das crianças resultou em uma melhora maior do que os informar sobre a precisão de seus resultados. Por ser mais demorada e cognitivamente mais exigente, nas matrizes maiores a estratégia de subtração foi menos utilizada. Gandini, Lemaire e Dufau (2008) buscaram compreender os processos de estimativa e suas mudanças com a idade. Os jovens foram quase sempre mais rápidos e mais precisos do que os adultos mais velhos, em cada estratégia.

Doze anos mais tarde que Siegler e Lemaire (1997) introduziram o método de escolha/não-escolha como um meio de obter características das estratégias cognitivas para realizar estimativas, Kovas e colaboradores (2009) examinaram a estimativa numérica de quantidades. Tinham como objetivo investigar os mecanismos cerebrais associados ao julgamento de quantidades e avaliar se as diferenças individuais na capacidade matemática estão associadas a esta tarefa. Os resultados sugeriram que, de forma semelhante aos adultos, múltiplas e distribuídas áreas cerebrais estão envolvidas na estimativa em crianças. E, apesar do desempenho comportamental igual, houve diferenças nos padrões de ativação cerebral entre os grupos de baixa e de alta capacidade matemática durante a tarefa, sugerindo que as diferenças individuais na capacidade matemática são refletidas na resposta do cérebro durante a solução da tarefa.

Para entender as estratégias de quantificação de itens e como elas são selecionadas, Gandini, Ardiale e Lemaire (2010) convidaram crianças a realizar estimativas de quantidades em coleções de 11 a 79 pontos, apresentados em diferentes configurações (aleatória e canônica). Embora Luwel e colaboradores (2000) tenham encontrado três estratégias principais no julgamento de quantidades (adição, subtração e estimativa rápida), Gandini, Ardiale e Lemaire (2010) revelaram seis estratégias: a) fixação: enumeração de alguns pontos (via contagem) e estimativa visual dos pontos restantes com base na primeira enumeração; b) referência: verificação do estímulo visual, recuperação de uma representação numérica na memória de longo prazo, comparação da diferença entre a representação codificada e a representação recuperada, e, em seguida, ajuste da sua resposta com base nesta diferença; c) decomposição/recomposição: separação em pequenos grupos de itens (cerca de 4), estimativa do número de grupos análogos e multiplicação destes resultados; d) aproximação de contagem: percepção aproximada de vários grupos de diferentes tamanhos adicionados, e) contagem exata: contagem de todos os pontos apresentados nas matrizes, adicionando sistematicamente todos os itens (um a um, dois a dois ou três a três) e f) outros: essas 
estratégias incluem relatos verbais que não correspondem a nenhuma das categorias anteriores.

Com foco ainda na escolha de estratégias, Schillemans e colaboradores (2009) realizaram dois experimentos em 31 adultos para testar se as escolhas estratégicas dos indivíduos em uma tarefa de julgamento de quantidades dispostas em matrizes são afetadas pela estratégia que foi utilizada nos testes anteriores. Para isso, os participantes deveriam resolver problemas, que envolviam grandes quantidades, com a estratégia de subtração e continuar a utilizar esta estratégia para quantidades neutras (nem grandes nem pequenas) e continuar a usá-la até que eles encontrassem uma quantidade de itens para a qual outra estratégia fosse claramente mais benéfica. Os resultados demonstraram que uma estratégia utilizada anteriormente influenciava as escolhas de estratégias posteriores, estando ela limitada aos itens que não têm uma forte associação com uma estratégia específica.

Em 2011, Schillemans e colaboradores (2011) forneceram evidência adicional para o efeito de "repetição da estratégia anterior" ocorrer mais frequentemente do que a mudança para outra estratégia. Nesta segunda ocasião, tendo que determinar o número de células coloridas em matrizes, os participantes tinham como possibilidade o uso da estratégia de adição ou da estratégia de subtração. As diferentes quantidades foram apresentadas em três ordens diferentes: 1) uma ordem ascendente [que começou com poucos itens (adição) e gradualmente aumentou para mais itens (subtração)], 2) uma ordem descendente (ordem inversa) e 3) uma ordem aleatória. Estes testes confirmaram a hipótese de que a quantidade de itens que exigia a troca da estratégia seria maior na ordem ascendente e menor na ordem descendente. Por outro lado, Schillemans e colaboradores (2011) não perceberam dados relevantes que comprovassem que a repetição de determinada estratégia utilizada em problema anterior seria utilizada de maneira mais frequente de que quando ela tivesse sido utilizada por mais de uma vez, em estratégias anteriores.

Como único estudo encontrado referente a estratégias utilizadas em tarefa de estimativa na reta numérica, Peeters, Verschaffel e Luwel (2016) identificaram que 63 adultos aplicavam espontaneamente estratégias baseadas em quartil, ao resolver uma tarefa de estimativa de Número-Posição e uma de Posição-Número na reta numérica 0-1000, na qual apenas a origem e o ponto final fossem indicados. Além disso, a precisão era maior e a variabilidade menor em números próximos das marcações, sendo a utilização de pontos de referência positivamente relacionada à precisão na reta numérica.

\section{Estudos de estimativa numérica de quantidades discretas}

Na perspectiva do desenvolvimento da estimativa numérica de quantidades, HuntleyFenner (2001) pediu a 15 crianças de 5 a 7 anos e adultos para realizarem estimativas das quantidades de 5, 7, 9 e 11 quadrados pretos, apresentados rapidamente e em distribuição aleatória, apontando para os números correspondentes em uma reta numérica que variava de 0 a 20, por 40 vezes cada quantidade, em 4 sessões. Os resultados indicaram estimativas semelhantes para crianças e adultos, exceto pelo fato do desvio padrão das estimativas 
diminuir com a idade. O que ficou claro a partir desses dados é que, embora os sentidos culturais (exatos e construídos através da linguagem) e analógicos (aproximados e independentes da linguagem) de número possam coexistir a partir do início do desenvolvimento, eles têm diferentes cursos no decorrer do desenvolvimento.

Também utilizando a tarefa de estimativa de quantidades, Lipton e Spelke (2005), encontraram uma relação linear entre as estimativas das crianças e a quantidade não simbólica real somente se a quantidade estava dentro do intervalo numérico ao qual a criança estivesse familiarizada. Em contraste, as crianças dificilmente discriminaram quantidade não simbólica que excederam a seu intervalo de familiaridade.

Surpreendentemente, em um estudo de estimativa numérica de quantidades de objetos em um recipiente, realizado por Booth e Siegler (2006), as crianças menores, da $1^{\text {a }}$ série, foram menos precisas que as da $2^{\mathrm{a}}$ série. Porém, ao contrário, as crianças da $3^{\mathrm{a}}$ série realizaram estimativas mais precisas que as da $4^{\mathrm{a}}$ série. Os pesquisadores concluíram que a estimativa de quantidades muda com a idade, paralelamente ao desenvolvimento de estimativas na reta numérica. No entanto, encontraram correlação positiva com $o$ desempenho em matemática para alunos da $3^{\mathrm{a}}$ e $4^{\mathrm{a}}$ séries, embora não para os alunos da $1^{\mathrm{a}} \mathrm{e}$ $2^{\mathrm{a}}$ séries.

Existem evidências de que crianças de apenas quatro anos de idade já são capazes de quantificar conjuntos e comparar essa quantidade à quantidade de outro conjunto. Por exemplo, no estudo de Jordan e colaboradores (2006), foram apresentados às crianças cartões com $3,8,15,25$ ou 35 pontos distribuídos ao acaso, de modo que o número de pontos aumentasse cada vez que fosse mostrado um novo cartão. A criança recebia uma pontuação correta se estimasse um valor com erro de até $25 \%$ do valor real, para mais ou para menos. Constatou-se que, antes mesmo de aprender aritmética convencional, crianças a partir dos 4 anos de idade podem realizar estimativa do tamanho de um conjunto de pontos: avaliar se um conjunto de pontos é maior ou menor do que um valor de referência indicado e julgar se um conjunto encaixa-se dentro de dois valores de referência (Jordan et al., 2006).

Para determinar se o desempenho em tarefas de quantificação é afetado por propriedades numéricas e perceptivas, Rousselle e Noel (2008) pediram a crianças de 3, 4, 5 e 6 anos para compararem quantidades (quantificação discreta) ou áreas (quantificação contínua) de coleções de pontos ou barras, variando ambas as dimensões. Os pesquisadores concluíram que a percepção de quantidades começa a determinar o processamento perceptivo aos 3 anos de idade e a sensibilidade aos estímulos numéricos aumenta com a idade. Da mesma forma, as propriedades perceptivas começam a interferir no processamento de quantidades com a mesma idade, mas estas influências permanecem bastante estáveis ao longo do desenvolvimento pré-escolar. Estes resultados sugerem que a automatização do processamento de quantidades surge gradualmente ao longo do desenvolvimento, ao passo que o acesso automático à informação perceptiva já está bem desenvolvido em pré-escolares.

Para verificar se o mapeamento espacial de números seria uma invenção cultural ou uma intuição universal compartilhada por todos os seres humanos, independentemente da 
cultura e da educação, Dehaene e colaboradores (2008) estudaram um grupo indígena da Amazônia (Mundurucus), de léxico numérico reduzido e pouca, ou nenhuma, educação formal. Os índios apresentaram representações logarítmicas para representações simbólicas e não simbólicas de números em todas as idades, enquanto que os adultos ocidentais utilizavam mapeamento linear com números pequenos ou simbólicos e mapeamento logarítmico com números não simbólicos. Os pesquisadores sugeriram que o mapeamento espacial de números é uma intuição universal e que este inicialmente é logarítmico.

Apoiando a hipótese da existência de um mecanismo dedicado a apreender pequenas quantidades diferente do que apreende quantidades maiores, Revkin e colaboradores (2008) realizaram um estudo com 18 adultos utilizando uma tarefa de estimativa de um conjunto de pontos em matrizes que variavam de 1 a 8 itens e de outro que variava de 10 a 80 itens. Várias medidas indicaram variabilidade nula ou muito pequena na faixa $1-4$, mas alta variabilidade e erros frequentes para quantidades de 10 a 40 nos conjuntos de 10 a 80 elementos.

Também encontrando relação entre a estimativa de quantidades e matemática, Gilmore, Mccarthy e Spelke (2010) realizaram testes padronizados de matemática em crianças de 5 anos de idade, bem como uma tarefa não-simbólica, em que as crianças observavam uma quantidade de pontos azuis adicionados a outra quantidade de pontos azuis e, então, relatavam se sua soma aproximada era mais ou menos numerosa do que uma quantidade de pontos vermelhos. A precisão das crianças na tarefa não-simbólica correlacionou-se com a sua habilidade matemática.

Na perspectiva de encontrar respostas sobre a representação numérica de magnitudes, Thompson e Siegler (2010) mediram estimativas de quantidades em matrizes de até 1000 pontos. As crianças observaram uma matriz vazia (zero pontos), outra cheia (1000 pontos) e uma terceira, inicialmente vazia, que deveria ser preenchida até que fosse alcançado o número desejado de pontos, utilizando-se as quantidades: "pequenas" $(5,18,53,79,164$, ou 237), "médias" (419, 487, 524, 548, 625, ou 632) ou "grandes" (725, 759, 817, 846, 938, ou 962). Neste experimento, as crianças, cuja estimativa global padrão era melhor ajustada pela função linear, utilizavam mais a representação logarítmica quando o número era inferior a 150 do que quando ele estava acima de 150.

Complementando os achados de Jordan e colaboradores (2006), Park e Brannon (2013) constaram que, mais do que quantificar conjuntos, crianças um pouco mais velhas podem ser capazes de operar com quantidades não numéricas. Os pesquisadores fizeram um estudo de intervenção apresentando aos participantes uma imagem de duas matrizes de pontos que continham entre 9 e 36 pontos cada. Em metade dos testes, os participantes foram solicitados a indicar se a soma ou a diferença entre os pontos nas duas matrizes era maior ou menor do que o número de pontos em uma terceira matriz. Na outra metade dos testes, os participantes foram solicitados a escolher qual das duas matrizes continha um número de pontos equivalente à soma ou à diferença entre o número de pontos nas duas matrizes 
inicialmente apresentadas. Os resultados mostraram que a melhora na tarefa de quantificação resultava em melhora na capacidade matemática simbólica.

Obersteiner e colaboradores (2014) compararam o desempenho de 202 crianças da $1^{\text {a }}$ série em dois tipos de tarefas informatizadas de enumeração de quantidades entre 1 e 20 pontos, apresentados em arranjos aleatórios ou em matriz. O número de pontos foi um forte preditor do tempo de resposta e das taxas de precisão na tarefa de enumeração com arranjos aleatórios, mas não foi preditor das respostas da tarefa com matriz. O desempenho na tarefa de matriz foi correlacionado com o desempenho em um teste de aritmética, mesmo quando outras variáveis cognitivas foram controladas.

Examinando as trajetórias de desenvolvimento de habilidades de processamento de magnitudes simbólicas e não simbólicas e como elas se relacionam entre si, Matejko e Ansari (2016) testaram 30 crianças do $1^{\circ}$ ano (quando as crianças estão se tornando mais fluentes com os números simbólicos). Os pesquisadores levantam a possibilidade de as trajetórias de desenvolvimento para o processamento simbólico e não simbólico mostrarem mais semelhanças no início do desenvolvimento. Por exemplo, as crianças entre 2 e 4 anos poderiam mostrar trajetórias de desenvolvimento semelhantes quando elas são expostas a símbolos numéricos pela primeira vez. As trajetórias mostraram também ter períodos de desenvolvimento diferentes, nos quais o processamento de magnitude não simbólica pode começar mais cedo e progredir mais lentamente do que o desenvolvimento de competências simbólicas. Ou seja, concluíram que as habilidades de processamento de magnitude simbólica e não simbólica tiveram trajetórias de desenvolvimento distintas.

\section{Estudos de estimativa na reta numérica}

Como já comentado anteriormente, uma tarefa amplamente utilizada para investigar como as pessoas representam números, é a Tarefa de Estimativa na Reta Numérica (Berteletti et al., 2010; Dehaene et al., 2008; Siegler \& Opfer, 2003). Nesta tarefa, os participantes são solicitados a posicionar um dado número em uma reta numérica vazia, que é delimitada por um valor inicial, geralmente zero, no início da linha, e outro valor, tal como 100 ou 1000, no final da reta numérica. Alguns pesquisadores têm argumentado que diferentes tipos de mudanças nas representações numéricas mentais podem implicar em mudanças no desempenho em estimativa na reta numérica. Corroborando a ideia de que as estimativas se tornam mais precisas com a idade, Siegler e Opfer (2003) constataram ainda que, com a idade, as estimativas das crianças de $1^{\mathrm{a}}, 4^{\mathrm{a}}$ e $6^{\mathrm{a}}$ séries alteraram substancialmente, especialmente na reta 0-1000. Além disso, as estimativas dos estudantes da $2^{\mathrm{a}}$ série encaixaram-se muito melhor no padrão logarítmico do que no linear. $\mathrm{Na} 4^{\mathrm{a}}$ série, as estimativas foram igualmente ajustadas pelos dois modelos. Em contrapartida, os alunos da $6^{\circ}$ série e os adultos tiveram suas estimativas melhor ajustadas ao modelo linear do que ao modelo logarítmico. A inclusão de um subconjunto de sete números em ambos os testes (0100 e 0-1000) permitiu aos pesquisadores constatar que a mesma criança pode representar o mesmo número de forma diferente, dependendo da faixa numérica. Além disso, a variação o 
tempo, de 4 para 30 segundos, não melhorou as estimativas.

Confirmando os resultados encontrados por Siegler e Opfer (2003), Siegler e Booth (2004) utilizaram a tarefa de estimativa na reta numérica 0-100 com 85 crianças, realizando dois experimentos em alunos pré-escolares e da $1^{\mathrm{a}}$ e $2^{\mathrm{a}}$ série acerca de suas representações internas. Concluíram que os padrões de estimativas progrediram de forma logarítmica (préescolares), a uma mistura de logarítmica e linear ( $1^{\text {a }}$ série) e, finalmente, a um padrão principalmente linear ( $2^{\mathrm{a}}$ série) e a linearidade foi responsável por melhora na precisão. A variabilidade das estimativas diminuiu com a idade, independente da magnitude numérica. As diferenças individuais na estimativa foram fortemente relacionadas com as diferenças individuais no desempenho em matemática, especialmente para a $1^{\mathrm{a}}$ e para a $2^{\mathrm{a}}$ séries. Replicando os resultados de Siegler e Opfer (2003), em que as estimativas das crianças tornaram-se mais lineares com a idade, na pesquisa de Opfer e Siegler (2007) utilizaram uma gama mais ampla de números a serem posicionados e encontraram resultados semelhantes.

A partir de 2008, crescem os estudos de estimativa numérica que utilizam a tarefa da reta numérica como instrumento de avaliação de desempenho nesta área. Preocupados, não tanto com a linearidade das estimativas, mas em compreender de que forma a estimativa estaria relacionada com o desempenho em aritmética, Booth e Siegler (2008) examinaram se a qualidade das representações de magnitude numérica dos alunos da $1^{\mathrm{a}}$ série (série em que em que, segundo os pesquisadores, há mais diferenças individuais na representação numérica entre 0 e 100) está relacionada de maneira preditiva com a aprendizagem da aritmética. Fizeram um teste de representações das magnitudes numéricas das crianças para correlacionar com o conhecimento aritmético. Verificaram que os resultados obtidos no teste foram capazes de predizer o desempenho em aritmética dos alunos. As relações entre as estimativas na reta numérica, a adição e os resultados dos testes de desempenho em matemática foram substanciais. Mesmo depois de os efeitos de outros aspectos do conhecimento numérico terem sido considerados, a linearidade das representações da magnitude numérica ainda previu a aprendizagem aritmética.

Da mesma maneira, Siegler e Mu (2008) verificaram que crianças pré-escolares $(\mathrm{N}=29)$ na China mostraram maior conhecimento numérico geral do que seus pares nos Estados Unidos ( $\mathrm{N}=24)$, não só para problemas de aritmética, mas também em estimativa na reta numérica, novidade para as crianças de ambos os países. As estimativas das crianças chinesas foram comparáveis às das crianças estadunidenses 1 a 2 anos mais avançadas na escola. As diferenças individuais na aritmética e o desempenho na tarefa da reta numérica foram correlacionados positivamente dentro de cada país. Ou seja, o maior conhecimento dos pré-escolares chineses e estadunidenses em aritmética parece melhorar a sua compreensão de magnitudes numéricas.

Na mesma perspectiva, Schneider, Grabner e Paetsch (2009) realizaram três estudos com um total de 429 alunos da $5^{\mathrm{a}}$ e $6^{\mathrm{a}}$ séries, concluindo que o conhecimento conceitual, a inteligência numérica e a estimativa na reta numérica foram bons preditores de desempenho matemático. Estes achados vão ao encontro dos resultados de Siegler e Booth (2004), de que 
as tarefas de estimativa e um teste padronizado de desempenho matemático estão correlacionados, mesmo após o controle da inteligência e da idade.

Ao contrário de estudos anteriores (Siegler \& Mu, 2008), no estudo de Muldoon e colaboradores (2011) com crianças chinesas $(\mathrm{N}=85)$ e escocesas $(\mathrm{N}=103)$, as crianças chinesas não apresentam escalas numéricas mais lineares antes que as escocesas e suas estimativas numéricas não foram mais precisas do que as da amostra escocesa de maior idade com habilidade matemática equivalente. Ambos os grupos de crianças eram capazes de contar até 10 e tinham conhecimento de números até 100.

Em especial, para discutir a forma como os números naturais são representados mentalmente, Berteletti e colaboradores (2010) investigaram o desenvolvimento da estimativa numérica em crianças de 3 a 6 anos de idade, usando retas numéricas 1-10, 1-20 e 0-100. Tinham como objetivo avaliar a capacidade das crianças de fornecer estimativas confiáveis (se logarítmica ou linear), tendo em vista que, a partir dos estudos anteriores de Siegler e Booth (2004), a mudança de logarítmica-linear já estaria estabelecida no contexto de 0-100 ou 0-1.000, mas não quando o intervalo numérico é restrito a unidades ou dezenas. Concluíram que as estimativas das crianças passaram de logarítmicas a lineares, sendo que elas se tornaram menos precisas, mas cada vez mais logarítmicas, no intervalo maior. A precisão da estimativa foi correlacionada com o conhecimento de algarismos arábicos e com a magnitude numérica.

Ainda para compreender a relação entre as representações numéricas e a matemática, Thompson e Siegler (2010) investigaram a relação entre as representações de magnitudes numéricas e memória de números em 18 crianças pré-escolares e alunos da $2^{\mathrm{a}}$ série, utilizando as tarefas das retas numéricas de 0-20 e de 0-1000. Os resultados indicaram que as representações de magnitude das crianças foram mais lineares para os números cujos nomes estão associados às suas grandezas (memória numérica superior).

Ao contrário desses achados, Barth e Paladino (2011) aplicaram modelos de raciocínio de proporcionalidade em estimativas na reta numérica 0-100 em crianças de 5 e 7 anos. A relação entre a posição estimada pelas crianças de 5 anos de idade e a posição real poderia ser descrita por uma adaptação do modelo de um ciclo. Este padrão sugere que as crianças de 5 anos usam a referência dos pontos inferior e superior como auxílio para suas estimativas. As estimativas das crianças de 7 anos, em contraste, eram melhor ajustadas por um modelo de dois-ciclos, o que implica que usavam também o ponto médio para realizar suas estimativas de maneira mais precisa. Esse ajuste dos modelos de representação das crianças para modelos de um ou dois ciclos sugere que estimativas podem ser baseadas numa estratégia de juízo proporcional em vez de refletir diretamente as características da reta numérica mental. Assim, a posição de um único número é estimada em relação à reta toda.

Para verificar estas conclusões, White e Szucs (2012) examinaram o uso de estratégias baseadas em ponto de referência em 67 crianças do $1^{\circ}$ ao $3^{\circ}$ ano em uma reta numérica 0-20 (intervalo numérico que já deveriam estar familiarizados). Os resultados sugeriram que as crianças do $1^{\circ}$ ano apenas utilizavam o ponto de referência inferior como uma orientação para 
as suas estimativas, tal como indicado pelo modelo logarítmico, gerando estimativas mais precisas e menos variáveis perto do ponto de referência mais baixo. As crianças do $2^{\circ}$ ano, em contraste, usaram também o ponto de referência superior para criar um ponto intermediário central, o que gerava estimativas menos variáveis e mais precisas para números também no meio do intervalo numérico, resultando num melhor ajuste do modelo linear. Esta estratégia tornou-se ainda mais evidente em crianças do $3^{\circ}$ ano.

Contradizendo a hipótese levantada por Siegler e Opfer (2003), Xu e colaboradores (2013) verificaram que a estimativa numérica pode não ser uma medida tão pura e independente do contexto, pois a cultura permite que as crianças sejam mais hábeis em realizar estimativas. Os pesquisadores (Xu et al., 2013) analisaram o desenvolvimento da estimativa numérica de 160 pré-escolares chineses de três grupos etários (3-4, 5 e 6 anos). Para isso, realizaram a tarefa de estimativa na reta numérica 0-10 com números, pontos e objetos e os três tipos de estímulos apresentaram o mesmo padrão de resultados. O grupo mais velho também realizou a atividade nas retas 0-100 e 0-1000. Concluíram que: a) a representação linear de números aumentou com a idade, mas que os chineses já são lineares desde muito cedo; b) a representação numérica foi consistente em todos os três tipos de tarefas; c) os participantes chineses geralmente utilizaram pontos de referência para atingir representações lineares e d) as estimativas de pré-escolares chineses mais velhos sobre as retas 0-100 e 0-1000 foram melhores do que nas demais, sendo o desempenho em matemática dos asiáticos sempre melhor, quando comparado aos seus pares ocidentais.

Evidências da correlação entre estimativa e habilidades numéricas também foram encontradas por Menzies e colaboradores (2013), em uma avaliação longitudinal. Os resultados mostraram que a qualidade das estimativas das crianças e o desempenho nas tarefas matemáticas melhoraram ao longo do mesmo período, mas as mudanças em uma não foram associadas a mudanças na outra. Em contraste com as alegações anteriores, de que a linearidade da representação numérica é uma contribuição única para o desenvolvimento matemático das crianças, os dados de Menzies e colaboradores (2013) sugeriram que essa variável não é significativamente privilegiada sobre habilidades numéricas básicas.

Abordando a questão da relação entre o desempenho matemático e habilidades espaciais, Lefevre e colaboradores (2013) coletaram um conjunto de dados longitudinais, durante 4 anos, de 101 crianças da $2^{\mathrm{a}}, 3^{\mathrm{a}}$ e $4^{\mathrm{a}}$ séries. Neste estudo, a capacidade espacial foi correlacionada com todas as medidas em matemática nos dois últimos anos, e com o desempenho da reta numérica do $3^{\circ}$ para o $4^{\circ}$ ano. No entanto, a capacidade espacial não previu um crescimento em aritmética ou sobre o sistema de numeração. Não foram encontradas evidências de que o desempenho na reta numérica é preditivo para cálculo mais do que o cálculo é preditivo no desempenho em estimativa na reta numérica. No entanto, o conhecimento do sistema de numeração no $3^{\circ}$ ano foi preditivo de desempenho na reta numérica no $4^{\circ}$ ano, independentemente da habilidade espacial. Sendo assim, a tarefa de estimativa na reta numérica não seria necessariamente um reflexo do sistema numérico mental. 
Contradizendo a afirmação de que o desempenho em comparação (maior/menor número) e em estimativa na reta numérica contém a mesma representação subjacente, Sasanguie e Reynvoet (2013) examinaram a relação entre o ajuste logarítmico-linear em uma tarefa de estimativa na reta numérica e o efeito do tamanho (números mais ou menos distantes entre si), em crianças da $1^{\mathrm{a}}, 2^{\mathrm{a}}$ e $3^{\mathrm{a}}$ séries. Nas duas experiências, não encontraram correlação entre estimativa na reta numérica e o efeito do tamanho. Também não encontraram diferença entre os grupos de crianças com uma representação linear ou logarítmica, considerando o efeito do tamanho. Os resultados sugeriram que diferentes mecanismos são subjacentes a ambas as tarefas básicas de processamento numérico.

Ebersbach, Luwel e Verschaffel (2015) avaliaram as habilidades de estimativa de 120 crianças, 40 pré-escolares, 40 da $1^{\mathrm{a}}$ série e 40 da $2^{\mathrm{a}}$ série, sobre uma tarefa de estimativa em retas numéricas tridimensionais delimitada e não-delimitada, considerando a familiaridade com números. Na condição de reta não-delimitada, apenas as posições de 1 e 10 foram marcadas. Na condição delimitada, a posição final 100 foi adicionalmente marcada. Os resultados indicaram que as estimativas foram mais precisas e menos variáveis em crianças que estavam familiarizadas com números maiores. A quantidade de pontos de referência não teve efeito de interação com familiaridade, destacando que a familiaridade das crianças com os números é um fator crucial para a qualidade das estimativas na reta numérica.

Em mais uma variação da tarefa de estimativa na reta numérica, Sasanguie e colaboradores (2016) apresentaram três considerações teóricas para o desenvolvimento de padrões de resposta em crianças da $1^{\mathrm{a}}, 2^{\mathrm{a}}$ e $6^{\mathrm{a}}$ séries, na tarefa de estimativa na reta numérica (simbólica e não simbólica): a mudança logaritmo-linear, a transformação de duplo-linear para linear e o juízo proporcional. O objetivo do estudo foi esclarecer qual modelo de representação melhor caracteriza o desenvolvimento dos padrões na reta numérica simbólica e não-simbólica. Observou-se que esses três modelos não foram contrastados. No caso de estimativas simbólicas, o julgamento proporcional descreveu melhor os dados. Na estimativa não-simbólica das crianças mais jovens, os padrões foram melhor descritos por um modelo logarítmico, enquanto que para as crianças mais velhas foram melhor descritos pelo modelo linear. Considerando que as crianças em idade escolar podem ser bem-sucedidas na reta numérica simbólica, a aplicação de tais estratégias ainda aparece muito difícil para as retas numéricas não-simbólicas, mesmo para as crianças mais velhas.

Mesmo com a vasta gama de estudos que utilizam a reta numérica como instrumento de medida da capacidade de realizar estimativas, pesquisadores revelaram que estimativas numéricas mapeadas em retas numéricas podem ser altamente intuitivas, visto que a criança facilmente percebe que números menores são localizados mais à esquerda da reta, enquanto números maiores mais à direita (Booth \& Siegler, 2006; Siegler \& Booth, 2004). Neste caso, as estimativas poderiam estar sendo alocadas de maneira mais intuitiva do que necessariamente pensadas em termos de escala pelas crianças. Lefevre e colaboradores (2013) sugerem cautela ao tirar conclusões sobre o desempenho da tarefa de estimativa na reta numérica como um reflexo das representações numéricas subjacentes das crianças. Essa relação também pode refletir a influência mútua do conhecimento conceitual das crianças 
sobre o sistema numérico e habilidades processuais para resolver um problema matemático, por exemplo.

\section{Considerações finais}

Evidencia-se que os estudos em estimativa numérica vêm sendo vastamente discutidos desde a década de 80, senão antes. Todos eles envolvem uma possível relação entre estimativa numérica e conhecimento matemático, tendo em vista que, este último tema, também já vem sendo pesquisado há bastante tempo. Mesmo assim, ainda pouco se sabe sobre como o sujeito (ou a criança) desenvolve o conhecimento matemático (ou a estimativa numérica), tendo em vista sua relevante importância para o sucesso na vida adulta. Em geral, os estudos em estimativa numérica discutem: a) as possíveis bases que originam esta habilidade, b) a representação mental das magnitudes numéricas, c) as maneiras de avaliar seu desempenho e de compará-lo ao desempenho matemático e d) o uso de estratégias de estimativa.

Quanto às possíveis bases cognitivas da estimativa numérica, pode-se destacar duas grandes correntes teóricas: a vertente inatista, que considera sua origem orgânica e inata, que evolui para a matemática formal. Ao contrário, a perspectiva construtivista entende a matemática como culturalmente construída e aprimorada em termos de complexidade com a idade e a experiência. Embora se entenda que a aprendizagem da matemática é progressiva, são considerados diversos fatores de bases orgânica que possibilitam este desenvolvimento posterior.

Os modelos de representação numérica mental vêm sendo desenvolvidos a partir de tarefas de estimativa na reta numérica. Primeiramente, um modelo mais elementar de representação logarítmica (Modelo Logarítmico) é proposto, para explicar o efeito da distância exagerada entre os números pequenos e minimizada para os grandes números. $\mathrm{O}$ Modelo Acumulador (ou Linear) propõe que a distância entre os números posicionados na reta numérica mental seria constante, independentemente da magnitude numérica estimada. Vários pesquisadores propuseram uma possível mudança de representação logarítmica-linear, a partir da idade, da experiência e do conhecimento numérico. Também é discutida a possibilidade de que as crianças possam obter as duas formas de representação concomitantemente, sendo testados o contexto numérico e a familiaridade das crianças com o intervalo numérico. Também foi proposto um modelo que considera duas representações lineares de inclinações distintas (Modelo Duplo-linear) e um Modelo de Juízo Proporcional, que considera o uso estratégico de estimativas com auxílio de pontos de referência conhecidos. Uma proposta integradora em que cada modelo possa ser considerado dependendo da etapa de desenvolvimento parece bastante plausível.

O desempenho em estimativa numérica vem sendo estudado através de duas tarefas conhecidas: a tarefa de estimativa na reta numérica e a tarefa de julgamento de quantidades discretas em um conjunto. Para realizar estimativa na reta numérica, é necessário representar um número em uma posição espacial ou vice-versa. Para realizar estimativa de quantidades, é 
necessário representar uma quantidade não-numérica (de itens) com um número. Em geral, os estudos sobre este tema discutem sobre a relação entre estimativa numérica e conhecimento numérico e/ou matemática. Em grande parte deles concluiu-se que a estimativa evolui com a idade, mas ainda não há consenso sobre uma correlação positiva da estimativa com o desempenho em matemática.

Sobre o uso de estratégias de estimativa numérica, os estudos apontaram diferentes perspectivas. Uma parte deles estava focada em compreender a mudança estratégica e outra, na enumeração de diferentes estratégias. Para essa segunda parte, ficou claro que as estratégias de soma e de subtração são as mais amplamente utilizadas, sendo que demais estratégias listadas podem estar dentre estas, como sendo uma situação particular, ou podem não ter sido encontradas em algumas pesquisas. Conclui-se que o tema da estimativa tem sido bastante estudado, mas os resultados das pesquisas ainda não são conclusivos.

\section{Referências}

Azevedo, M. M. D. O. (2016). A Aprendizagem da Estimação Matemática: um estudo no $2^{\circ}$ ciclo. Dissertação de Mestrado. Lisboa: Universidade Nova de Lisboa.

Barth, H. C. \& Paladino, A. M. (2011). The Development of Numerical Estimation: evidence against a representational shift. Developmental Science, 14, 125- 135.

Berch, D. B. (2005). Making Sense of Number Sense. Journal of Learning Disabilities, 38(4), 333-339.

Berteletti, I. et al. (2010). Numerical Estimation in Preschoolers. Developmental Psychology, 46 (2), 545-551.

Booth, J. L. \& Siegler, R. S. (2006). Developmental and Individual Differences in Pure Numerical Estimation. Developmental Psychology, 41, 189-201.

Booth, J. L. \& Siegler, R. S. (2008). Numerical Magnitude Representations Influence Arithmetic Learning. Child Development, 79 (4), 1016 - 1031.

Corso, L. V. \& Dorneles, B. V. (2010). Senso Numérico e Dificuldades de Aprendizagem na Matemática. Revista Psicopedagogia, 27, 298-309.

Crites, T. W. (1992). Skilled and Less Skilled Estimators' Strategies for Estimating Discrete Quantities. Elementary School Journal, 5, 601-619.

Dackermann, T. et al. (2015). An Integration of Competing Accounts on Children's Number Line Estimation. Frontiers in Psychology, 6 (884).

Dehaene, S. (1997). The Number Sense: How the mind creates mathematics. New York: Oxford University Press.

Dehaene, S. et al. (2008). Log or Linear? Distinct Intuitions of the Number Scale in Western and Amazonian Indigene Cultures. Stanislas Science, 320, 1217.

Dorneles, B. V. et al. (2017). Number Estimation in Children: an assessment study with number line estimation and numerosity tasks. In Congress of the European Society for Research in Mathematics Education (CERME), 10., 2017, Dublin City University. 
Ebersbach, M. et al. (2008). The Relationship Between the Shape of the Mental Number Line and Familiarity with Numbers in 5- to 9-Year Old Children: Evidence for a segmented linear model. Journal of Experimental Child Psychology, 99, 1-17.

Ebersbach, M., Luwel, K. \& Verschaffel, L. (2015). The Relationship Between Children's Familiarity with Numbers and Their Performance in Bounded and Unbounded Number Line Estimations. Mathematical Thinking and Learning, 17, 136-154.

Feigenson, L., Libertus, M. E. \& Halberda, J. (2013). Links Between the Intuitive Sense of Number and Formal Mathematics Ability. Child Development Perspectives, 7 (2), 74-79.

Gandini, D., Ardiale, E.\& Lemaire, P. (2010). Children' Strategies in Approximate Quantification. Current Psychology Letters: Behaviour, Brain, \& Cognition, 26, 114.

Gandini, D., Lemaire, P. \& Dufau, F. (2008). Older and Younger Adults' Strategies in Approximate Quantification. Acta Psychologica, 129, 175-189.

Gebuis, T. \& Reynvoet, B. (2012). The Role of Visual Information in Numerosity Estimation. PLoS One, 7(5).

Gibbon, J. \& Church, R. M. (1981). Time Left: Linear Versus Logarithmic Subjective Time. Journal of Experimental Psychology: Animal Behavior Processes, 7(2), 87108.

Gilmore, C., Mccarthy, S. \& Spelke, E. (2010). Non-symbolic Arithmetic Abilities and Achievement in the First Year of Formal Schooling in Mathematics. Cognition, 115(3), 394-406.

Halberda, J. \& Feigenson, L. (2008). Developmental Change in the Acuity of the "Number Sense": the approximate number system in 3, 4, 5, and 6-year-olds and adults. Developmental Psychology, 44, 1457-1465.

Huntley-Fenner, G. (2001). Children's Understanding of Number is Similar to Adults' and Rats': numerical estimation by 5 7-year-olds. Cognition, 78, B27-B40.

Jordan, N. C. et al. (2006). Number Sense Growth in Kindergarten: a longitudinal investigation of children at risk of mathematics difficulties. Child Development, 77, 153-175.

Kovas, Y., Giampietro, V., Viding, E., Ng, V., Brammer, M., Barker, G. J., Happe, F. G. E. \& Plomin, R. (2009). Brain Correlates of Non-Symbolic Numerosity Estimation in Low and High Mathematical Ability Children. PlosOne, 4(2).

Laski, E. V. \& Siegler, R. S. (2007). Is 27 a Big Number? Correlational and Causal Connections Among Numerical Categorization, Number Line Estimation, and Numerical Magnitude Comparison. Child Development, 78 (6), 1723 - 1743.

Lefevre, J. et al. (2013). Charting the Role of the Number Line in Mathematical Development. Front. Psychol, 4.

Lemaire, P. \& Siegler, R. (1995). Four Aspects of Strategic Change: contributions to children's learning of multiplication. Journal of Experimental Psychology: General, 124 (1), 83-97. 
DOI: $10.20396 /$ zet.v27i0.8652389

Levine, D. R. (1982). Strategy Use and Estimation Ability of College Students. Journal for Research in Mathematics Education, 13, 350-359.

Libertus, M., Feigenson, L. \& Halberda, J. (2011). Preschool Acuity of the Approximate Number System Correlates with School Math Ability. Developmental Science, 14:6, 1292-1300.

Link, T., Nuerk, H. \& Moeller, K. (2014). On the Relation Between the Mental Number Line and Arithmetic Competencies. The Quarterly Journal of Experimental Psychology, 67:8, 1597-1613.

Lipton, J. S. \& Spelke, E. S. (2005). Preschool Children's Mapping of Number Words to Nonsymbolic Numerosities. Child Development, 76:5, 978 - 988.

Luwel, K. et al. (2001). Using Segmented Linear Regression Models with Unknown Change Points to Analyze Strategy Shifts in Cognitive Tasks. Behavior Research Methods, Instruments, \& Computers, 33 (4), 470-478.

Luwel, K. et al. (2009). Strengths and Weaknesses of the Choice/No-Choice Method in Research on Strategy Use. European Psychologist, 14(4), 351-362.

Luwel, K. \& Verschaffel, L. (2003). Adapting Strategy Choices to Situational Factors: the effect of time pressure on children's numerosity judgement strategies. Psychologica Belgica, 43, 269-295.

Luwel, K. \& Verschaffel, L. (2008). Analyzing Strategy Use in Terms of the Four Parameters of Strategic Competence: contributions from a numerosity judgment task. Anales de Psicología, 24(2), 223-239.

Luwel, K. et al. (2000). Children's Strategies for Numerosity Judgment in Square Grids of Different Sizes. Psychologica Belgica, 40 (3), 183-209.

Matejko, A. A. \& Ansari, D. (2016). Trajectories of Symbolic and Nonsymbolic Magnitude Processing in the First Year of Formal Schooling. PlosOne, 1-15.

Menzies, V. et al. (2013). A Longitudinal Analysis of Estimation, Counting Skills, and Mathematical Ability Across the First School Year. Developmental Psychology, 49 (2), 250-257.

Moeller, K. et al. (2009). Children's Early Mental Number Line: Logarithmic or decomposed linear? Journal of Experimental Child Psychology, 103 (4), 503-515.

Muldoon, K. et al. (2011). Cross-Cultural Comparisons of 5-Year-Olds' Estimating and Mathematical Ability. Journal of Cross-Cultural Psychology, 42(4), 669-681.

Nuerk, H. C. et al. (2004). On the Development of the Mental Number Line: More, Less, or Never Holistic with Increasing Age? Developmental Psychology, 40(6), 11991211.

Obersteiner, A. et al. (2014). Do First Graders Make Efficient Use of External Number Representations? The Case of the Twenty-Frame. Cognition and Instruction, 32(4), 353-373.

Opfer, J. \& Siegler, R. (2007). Representational change and children's numerical estimation. Cognitive Psychology, 55, 169-195. 
Park, J. \& Brannon, E. M. (2013). Training the Approximate Number System Improves Math Proficiency. Psychol Sci, 24(10), 2013-9.

Peeters, D., Verschaffel, L. \& Luwel, K. (2016). Benchmark-Based Strategies in Whole Number Line Estimation. British Journal of Psychology.

Revkin, S. K. et al. (2008). Does Subitizing Reflect Numerical Estimation? Psychological Science, 19(6), 607-614.

Rousselle, L. \& Noel, M. P. (2008). The Development of Automatic Numerosity Processing in Preschoolers: evidence for numerosity-perceptual interference. Developmental Psychology, 44(2), 544-560.

Sasanguie, D. \& Reynvoet, B. (2013). Number Comparison and Number Line Estimation Rely on Different Mechanisms. Psychologica Belgica, 53:4, 17-35.

Sasanguie, D. et al. (2016). The Development of Symbolic and Non-Symbolic Number Line Estimations: three developmental accounts contrasted within cross-sectional and longitudinal data. Psychologica Belgica, 56(4), 382-405.

Schillemans, V. et al. (2009). The Influence of Previous Strategy Use on Individuals' Subsequent Strategy Choice: findings from a numerosity judgement task. Psychologica Belgica, 49(4), 191-205.

Schillemans, V. et al. (2011). The Influence of the Previous Strategy on Individuals' Strategy Choices. Studia psychologica, 53(4), 339-350.

Schneider, M., Grabner, R. H. \& Paetsch, J. (2009). Mental Number Line, Number Line Estimation, and Mathematical Achievement: their interrelations in grades 5 and 6. Journal of Educational Psychology, 101, 359-372.

Siegel, A. W., Goldsmith, T. H. \& Madson, C. R. (1982). Skill in Estimation Problems of Extent and Numerosity. Journal for Research in Mathematics Education, 13, 211232.

Siegler, R. S. \& Booth, J. L. (2004). Development of Numerical Estimation in Young Children. Child Development, 75, 428-444.

Siegler, R. \& Booth, J. L. (2005). Development of Numerical Estimation: a review. In Campbell, J. I. D. (2005). Handbook of Mathematical Cognition. Psychology Press: New York. Cap. 2, p. 197-212.

Siegler, R. \& Lemaire, P. (1997). Older and Younger Adult's Strategy Choices in Multiplication: testing predictions of ASCM using the choice/no-choice method. Journal of Experimental Psychology: General, 126 (1), 71-92.

Siegler, R. S. \& Mu, Y. (2008). Chinese Children Excel on Novel Mathematics Problems Even Before Elementary School. Psychological Science, 19, 759-763.

Siegler, R. S. \& Opfer, J. E. (2003). The Development of Numerical Estimation: evidence for multiple representations of numerical quantity. Psychological Science, $14,237-243$.

Starkey, P. \& Cooper, R. G. (1980). Perception of Numbers by Human Infants. Science, 210, 1033-1035. 
DOI: $10.20396 /$ zet.v27i0.8652389

Thompson, C. A. \& Siegler, R. S. (2010). Linear Numerical-Magnitude Representations Aid Children's Memory for Numbers. Psychological Science, 21(9), 1274-1281.

Verschaffel, L. et al. (1998). The Acquisition and Use of an Adaptive Strategy for Estimating Numerosity. European Journal of Psychology of Educuation, 13, 347370 .

White, S. L. J. \& SzüC, D. (2012). Representational Change and Strategy Use in Children's Number Line Estimation During the First Years of Primary School. Behavioral and Brain Functions, 8:1.

$\mathrm{Xu}, \mathrm{X}$. et al. (2013). Development of Numerical Estimation in Chinese Preschool Children. Journal of Experimental Child Psychology, 116, 351-366. 